\title{
The Genome of the Netherlands: design, and project goals
}

Dorret I Boomsma ${ }^{\star, 1,22}$, Cisca Wijmenga ${ }^{2,22}$, Eline P Slagboom ${ }^{3,22}$, Morris A Swertz ${ }^{2,22}$, Lennart C Karssen ${ }^{4}$, Abdel Abdellaoui ${ }^{1}$, Kai Ye ${ }^{3}$, Victor Guryev ${ }^{5,6}$, Martijn Vermaat ${ }^{7,8,9}$, Freerk van Dijk ${ }^{2}$, Laurent C Francioli ${ }^{10}$, Jouke Jan Hottenga ${ }^{1}$, Jeroen FJ Laros ${ }^{7,89}$, Qibin $\mathrm{Li}^{11}$, Yingrui $\mathrm{Li}^{11}$, Hongzhi Cao ${ }^{11}$, Ruoyan Chen ${ }^{11}$, Yuanping Du ${ }^{11}$, Ning Li ${ }^{12}$, Sujie Cao ${ }^{12}$, Jessica van Setten ${ }^{10}$, Androniki Menelaou ${ }^{10}$, Sara L Pulit ${ }^{10}$, Jayne Y Hehir-Kwa ${ }^{15}$, Marian Beekman ${ }^{16}$, Clara C Elbers ${ }^{10}$, Heorhiy Byelas ${ }^{2}$, Anton JM de Craen ${ }^{16}$, Patrick Deelen ${ }^{2}$, Martijn Dijkstra ${ }^{2}$, Johan T den Dunnen ${ }^{8,9}$, Peter de Knijff ${ }^{8,9}$, Jeanine Houwing-Duistermaat ${ }^{17}$, Vyacheslav Koval ${ }^{18}$, Karol Estrada ${ }^{18}$, Albert Hofman ${ }^{4}$, Alexandros Kanterakis ${ }^{2}$, David van Enckevort ${ }^{7}$, Hailiang Mai ${ }^{7}$, Mathijs Kattenberg ${ }^{1}$, Elisabeth $M$ van Leeuwen ${ }^{4}$, Pieter BT Neerincx ${ }^{2}$, Ben Oostra ${ }^{19}$, Fernanodo Rivadeneira ${ }^{18}$, Eka HD Suchiman ${ }^{3}$, Andre G Uitterlinden ${ }^{18}$, Gonneke Willemsen ${ }^{1}$, Bruce H Wolffenbuttel ${ }^{20}$, Jun Wang ${ }^{11,13,14,22}$, Paul IW de Bakker ${ }^{10,22}$, Gert-Jan van Ommen ${ }^{21,22}$ and Cornelia M van Duijn ${ }^{\star 4,22}$

Within the Netherlands a national network of biobanks has been established (Biobanking and Biomolecular Research Infrastructure-Netherlands (BBMRI-NL)) as a national node of the European BBMRI. One of the aims of BBMRI-NL is to enrich biobanks with different types of molecular and phenotype data. Here, we describe the Genome of the Netherlands (GoNL), one of the projects within BBMRI-NL. GoNL is a whole-genome-sequencing project in a representative sample consisting of 250 trio-families from all provinces in the Netherlands, which aims to characterize DNA sequence variation in the Dutch population. The parent-offspring trios include adult individuals ranging in age from 19 to 87 years (mean $=53$ years; $S D=16$ years) from birth cohorts 1910-1994. Sequencing was done on blood-derived DNA from uncultured cells and accomplished coverage was 14-15x. The family-based design represents a unique resource to assess the frequency of regional variants, accurately reconstruct haplotypes by family-based phasing, characterize short indels and complex structural variants, and establish the rate of de novo mutational events. GoNL will also serve as a reference panel for imputation in the available genome-wide association studies in Dutch and other cohorts to refine association signals and uncover population-specific variants. GoNL will create a catalog of human genetic variation in this sample that is uniquely characterized with respect to micro-geographic location and a wide range of phenotypes. The resource will be made available to the research and medical community to guide the interpretation of sequencing projects. The present paper summarizes the global characteristics of the project. European Journal of Human Genetics (2014) 22, 221-227; doi:10.1038/ejhg.2013.118; published online 29 May 2013

Keywords: whole-genome sequence; trio-design; population genetics

\section{INTRODUCTION}

The last decade has seen rapid developments and breakthroughs in unraveling the genetic etiology of complex genetic disorders. ${ }^{1}$ Genome-wide association studies (GWAS) have uncovered thousands of variants influencing major diseases and complex human traits, including cardiovascular disease, diabetes, dementia, schizophrenia, height, and body mass index (BMI). Despite major successes in gene discovery, for many traits the percentage of variance

\footnotetext{
${ }^{1}$ Department of Biological Psychology, VU University Amsterdam, Netherlands Twin Register, Amsterdam, The Netherlands; ${ }^{2}$ University of Groningen, University Medical Center Groningen, Department of Genetics, Groningen, The Netherlands; ${ }^{3}$ Molecular Epidemiology Section, Leiden University Medical Center, Netherlands Consortium for Healthy Ageing, Leiden, The Netherlands; ${ }^{4}$ Department of Epidemiology, Erasmus Medical Center, Rotterdam, The Netherlands; ${ }^{5}$ European Research Institute for the Biology of Ageing, University Medical Center Groningen, University of Groningen, Groningen, The Netherlands; ${ }^{6}$ Hubrecht Institute, Royal Netherlands Academy of Arts and Sciences, University Medical Center Utrecht, Utrecht, The Netherlands; ${ }^{7}$ Netherlands Bioinformatics Centre, Nijmegen, The Netherlands; ${ }^{8}$ Department of Human Genetics, Center for Human and Clinical Genetics, Leiden University Medical Center, Leiden, The Netherlands; ${ }^{2}$ Leiden Genome Technology Center, Leiden University Medical Center, Leiden, The Netherlands; ${ }^{10}$ Department of Medical Genetics, University Medical Center Utrecht, Utrecht, The Netherlands; ${ }^{11}$ BGI-Shenzhen, Shenzhen, China; ${ }^{12}$ BGI-Europe, Copenhagen, Denmark; ${ }^{13}$ Department of Biology, University of Copenhagen, Copenhagen, Denmark; ${ }^{14}$ The Novo Nordisk Foundation Center for Basic Metabolic Research, University of Copenhagen, Copenhagen, Denmark; ${ }^{15}$ Department of Human Genetics, Radboud University Medical Centre, Nijmegen, The Netherlands; ${ }^{16}$ Department of Gerontology and Geriatrics, Leiden University Medical Centre, Leiden, The Netherlands; ${ }^{17}$ Department of Medical Statistics and Bioinformatics, Leiden University Medical Centre, Leiden, The Netherlands; ${ }^{18}$ Erasmus Medical Centre, Genetic Laboratory Internal Medicine, Rotterdam, The Netherlands; ${ }^{19}$ Department of Clinical Genetics, Erasmus University Medical School, Rotterdam, The Netherlands; ${ }^{20}$ LifeLines Cohort Study \& Department of Endocrinology, University Medical Center Groningen, University of Groningen, Groningen, The Netherlands; ${ }^{21}$ Department of Human Genetics, Leiden University Medical Centre, Leiden, The Netherlands

22These authors contributed equally to this work.

${ }^{*}$ Correspondence: Professor Dr DI Boomsma, Department of Biological Psychology, VU University Amsterdam, Netherlands Twin Register, Van der Boechorststraat 1, 1081 BT Amsterdam, The Netherlands. Tel: + 3120598 8787; Fax: + 3120598 8832; E-mail: di.boomsma@vu.nl.

or Professor Dr CM van Duijn, Department of Epidemiology, Erasmus Medical Center, PO Box 2040, 3000 CA Rotterdam, The Netherlands. Tel: + 31 10 7043394 ; Fax: + 31107044 657; E-mail: c.vanduijn@erasmusmc.nl

Received 7 December 2012; revised 28 February 2013; accepted 24 March 2013; published online 29 May 2013
} 
explained by GWAS loci is relatively low, leaving a substantial part of the variation to be explained. Approaches that estimate genetic variance based on a large series of GWAS variants such as GCTA $^{2,3}$ show that aggregate effects of genome-wide significant common variants and those that do not reach this criterion explain substantially larger amounts of variance for, for example, human height, ${ }^{4}$ major depression ${ }^{5}$ or type 1 and 2 diabetes. ${ }^{6}$ Clearly, GWAS have not yet reached their limits, as is demonstrated by the many new loci detected after increasing sample sizes.

The use of dedicated arrays such as the metaboChip and immunoChip, which include new common and rare variants from the established GWAS regions, confirm the findings of modeling studies that the heritability not yet explained is to be found in a mixture of unmapped and untagged common, and not yet discovered rare variants. ${ }^{7-10}$ Despite progress, our knowledge of the regions identified by GWAS is still limited. Considering that even for 'simple' genetic diseases such as cystic fibrosis thousands of relevant mutations have occurred in a single gene, there is a need for in-depth sequencing and characterization of the genomic regions identified thus far. In a selected, relatively small, group of 256 Sardinians with extreme LDL-C values who were resequenced and reimputed to the 1000 Genomes Project reference at six GWAS loci, the variance explained for blood lipid levels at known loci doubled through the identification of both rare and common variants. ${ }^{11}$

Thus, identifying low frequency and rare variants by resequencing will contribute to resolving the missing heritability. As rarer variants are more likely to be population specific, ${ }^{12}$ sequencing across a range of different populations is mandatory for translation into public health and clinical benefits. One aim of the 1000 Genomes Project is to create maps of genetic variation across multiple populations, but the number of individuals per population is modest. Tennessen et al $l^{12}$ identified $>500000$ single-nucleotide variants (SNVs) in a sequencing project of 15585 human protein-coding genes. The major part of these SNVs were rare $(86 \%$ with a minor allele frequency $<0.5 \%)$, unknown $(82 \%)$ and population-specific $(82 \%)$. A deep-sequencing study ${ }^{13}$ of 202 putative drug targets confirmed that most genetic variants occur at very low frequencies. To characterize a population, it is crucial to sequence as many individuals as possible to maximize the probability of capturing rare variants.

Findings from sequencing studies are also important for clinical applications and public health genetics. The interpretation of the 'potential causality' of a novel variant after it has been identified in patients in a clinical setting, requires knowledge of its frequency in the population the patient belonged to. In view of its potential research and clinical applications, the Genome of the Netherlands (GoNL; http://www.nlgenome.nl) project was initiated as a part of BBMRI$\mathrm{NL}^{14}$ (Biobanking and Biomolecular Research InfrastructureNetherlands). The aim of GoNL was to sequence 1000 independent genomes from the Dutch population. These 1000 Genomes come from a representative sample of 250 parent-pairs with 1 or 2 offspring. Sequencing was done using genomic DNA to an intended average depth of $12 x$ for each sample. Here, we describe the design of GoNL within the demographic setting of the Netherlands, the sampling frame and basic characteristics of the participants.

Geographically, the Netherlands is a small country of about $41500 \mathrm{~km}^{2}$. Yet, the Dutch population of nearly 17 million inhabitants constitutes a middle-sized population within Europe and represents 0.24 percent of the world's total population. The Netherlands were populated by different groups. ${ }^{15,16}$ Regional isolation and religious endogamy, as well as rapid population growth had key roles in shaping the population genetics of the Dutch. Population growth was low until the 19th century, and doubled between 1900 and 1950 from 5.1 to 10 million people and between 1951 and 2000 to 15.9 million. In 1901, the total fertility rate was 4.53 , in 1950 it was 3.10 and in 2000 it had decreased to 1.72. From the 1970s onwards (http:// statline.cbs.nl/StatWeb/publication), the number of non-western immigrants increased from $\sim 1.2 \%$ of the population to $10.4 \%$ in 2005 and is now stable at $11.6 \%$. Immigrants have been of various genetic backgrounds, with Turkish and Moroccan origin being the largest groups followed by those of Asian and African origin from (former) Dutch overseas areas (Netherlands Antilles, Suriname and Indonesia). At the design stage of the GoNL project, we decided to include participants whose ancestors are born in the Netherlands. Sequencing of other groups was not possible without compromising the power for all the groups. However, extending sequencing beyond Caucasians remains a desirable aim.

Another key decision concerned the selection of subjects over geographic regions. The Netherlands are historically divided into provinces, which are geographical units with their own governance dating back from the period that the Netherlands were a republic (1588-1795). Geographically, the distribution of the population in the Netherlands is heavily skewed with the majority $(75 \%)$ of the population living in the Western and Middle provinces of the country including North-Holland $(16 \%$ of the population including the capital Amsterdam), South-Holland (22\% including the largest European harbor, Rotterdam), North-Brabant (15\%, bordering Belgium, formerly part of the Dutch kingdom), Gelderland (12\%, bordering Germany) and Utrecht (10\%; see Table 1 and Figure 1); only a small fraction lives in the Northern and Southern provinces. There is considerable evidence for regional differences in genetic make-up, for example, there is a significant North-South gradient both in height and eye color. ${ }^{15}$ Also studies of the Y-chromosome clearly pinpoint differences in genetic origin.

Representative sampling from the provinces of the Netherlands according to sample size would lead to major differences in numbers of subjects sequenced per region. Although this reflects the size and distribution of the population, many population genetic analyses are influenced by sample size, including linkage disequilibrium and founder effects. We, therefore, chose to include an approximately

Table 1 Participants by province of Netherlands, and \% of the population in that region

\begin{tabular}{|c|c|c|c|c|}
\hline Province & $\begin{array}{c}\text { Size of population } \\
\text { relative to total } \\
\text { (\% to total of } 15-65 \\
\text { years) }\end{array}$ & $\begin{array}{l}\text { Number of } \\
\text { subjects } \\
\text { included } \\
\text { in GoNL }\end{array}$ & $\begin{array}{c}\% \text { of the } \\
\text { GoNL } \\
\text { sample }\end{array}$ & $\begin{array}{l}\text { Number of } \\
\text { twin pairs }\end{array}$ \\
\hline Drenthe & 2.8 & 56 & 7.3 & \\
\hline Friesland & 3.8 & 62 & 8.1 & \\
\hline Gelderland & 11.9 & 57 & 7.4 & $4 \mathrm{MZ}, 2 \mathrm{DZ}$ \\
\hline Groningen & 3.5 & 57 & 7.4 & \\
\hline Limburg & 6.7 & 58 & 7.5 & $1 \mathrm{MZ}, 2 \mathrm{DZ}$ \\
\hline North-Brabant & 14.8 & 68 & 8.8 & $1 \mathrm{MZ}$ \\
\hline North-Holland & 16.5 & 91 & 11.8 & $1 \mathrm{MZ}$ \\
\hline Overijssel & 6.7 & 58 & 7.5 & $2 \mathrm{MZ}, 2 \mathrm{DZ}$ \\
\hline Utrecht & 9.5 & 48 & 6.2 & $2 \mathrm{MZ}$ \\
\hline Zeeland & 2.2 & 46 & 6.0 & $2 \mathrm{DZ}$ \\
\hline $\begin{array}{l}\text { South- } \\
\text { Holland }\end{array}$ & 21.5 & 168 & 21.8 & \\
\hline Total & & 769 & & 19 Twin pairs \\
\hline
\end{tabular}

Abbreviations: MZ monozygotic, DZ dizygotic. 


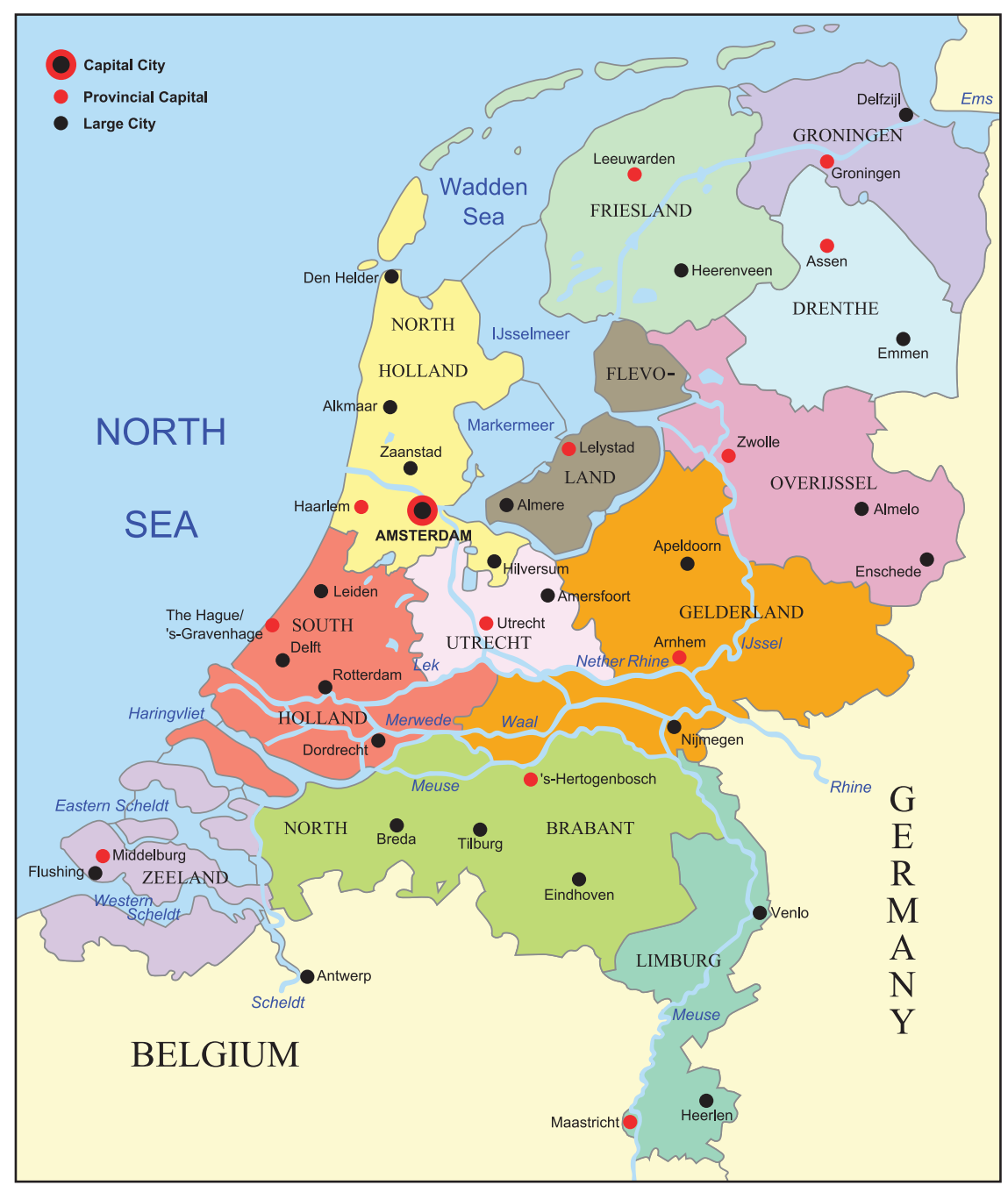

Figure 1 The 12 provinces of the Netherlands, the 12th province (Flevoland) is a recent province (land reclaimed from water) and was not included as a separate sampling unit (Image by Wikimedia Commons user Alphathon).

equal representation from the original 11 Dutch provinces (see Table 1), oversampling the two major cities Amsterdam and Rotterdam. The 12th province, which was established in 1986, was not included, as it was populated by recent immigration from surrounding areas.

\section{Trio design}

A key characteristic of GoNL is its trio design. The sample unit consists of two parents and one offspring yielding information on four genomes (two per parent). There were three considerations that led to the choice of the trio design for GoNL despite the fact that a trio design reduces the effective sample size by one-third compared with a design that includes only unrelated individuals. First, a major aim is to provide a facility for imputation, rapid and valid haplotyping, and the trio design allowed GoNL to reduce the computational burden of haplotyping. Second, a trio design would enable reducing the number of false-positive variant calls by screening for violation of Mendelian segregation in the trio-aware calling of genotypes. Third, the trio design would allow for a characterization of new mutations within the Dutch population, in that novel variants that occur in offspring but not in parents can be assessed. This latter approach requires a separate calling and storage of variants in which
non-Mendelian transmission is allowed. Our sequencing at intermediate coverage was considered to be optimal to address these aims.

\section{Selection of samples}

In the Netherlands, several large-scale collaborative Biobank consortia have been established, that bring together patient samples and population-based collections of biological samples. GoNL is part of the Dutch branch of BBMRI (http://www.bbmri.nl/). In BBMRI-NL, ${ }^{14}$ $\sim 180$ biobanks from all over the Netherlands have been united, including over 600000 subjects with biological samples and phenotype information. For 100000 to 150000 subjects genome-wide SNP data are available, or are currently being generated. For the GoNL trio study, four population-based biobanks provided samples:

1. LifeLines (http://lifelines.nl/lifelines-research/general), which is a three-generation population-based cohort now including more than 100000 participants (target 160000 ) representing $\sim 10 \%$ of the northern part of the Netherlands. ${ }^{17}$

2. The Leiden Longevity Study (http://www.healthy-ageing.nl; http://www.langleven.net), which includes 3500 persons from families in which at least two long-lived siblings were alive and their parents were of Caucasian descent. ${ }^{18}$ 
3. The Netherlands Twin Registry (NTR: http://www.tweelingenregister. org), which ascertains two- and three-generation Dutch families from the entire country based on the presence of twins or higher order multiples in the family and includes nearly 180000 participants. ${ }^{19,20}$ The NTR Biobank collected biological samples in $>10000$ participants. ${ }^{21}$

4. The Rotterdam studies, which consist of a population-based longterm follow-up study including 11800 persons from Rotterdam ${ }^{22}$ and its surrounding area (http://www.erasmus-epidemiology.nl/ rotterdamstudy) and the Genetic Research in Isolated Populations program (GRIP: http://www.epib.nl/research/geneticepi/research. html\#gip) targeting the south western part of the Province North-Brabant.

Table 1 summarizes the geographical distribution of the GoNL participants over the Netherlands. A total of 769 subjects were included from 250 families. In 19 of the 250 families, 2 offspring were sequenced, 11 monozygotic (MZ) and 8 dizyotic (DZ) twin pairs (see Figure 2). All participants are part of active biobanks. For the majority of participants information on their place of birth and place of birth of their (grand) parents is known. As the participants come from prospective studies, a wealth of phenotype data is available, including risk factors for morbidity and mortality, and information on lifestyle and medication use. As part of BBMRI-NL, these data will be further enriched with metabolomics (NMR, mass spectrometry), whole-genome epigenetic (450 K Illumina methylation arrays) and RNA-sequencing data. Information on morbidity is collected by record linkage projects in the Netherlands with databases such as PALGA (The nationwide network and registry of histo- and cytopathology in the Netherlands), NKR (Netherlands Cancer Registration) and others.

Table 2 provides the demographic background of the sample. The mean maternal age at blood draw was 62 years (SD 8.4 years), the mean paternal age 64 (SD 8.6 years) and that of the offspring 36 (SD
9.3 years). On average there is a $5 \mathrm{~cm}$ increase in height in the second generation, as is consistent with general population data. Average BMI values show the population to be relatively healthy, although persons with severe obesity are also observed. Lipid levels are relatively low, in line with the low BMI and the relatively high percentage of lipid lowering medication users in the Dutch population.

\section{Sequencing}

A coverage of $12 \mathrm{x}$ in both parents and the offspring was chosen based on balancing sensitivity (calling completeness) and accuracy (erroneous false-positive variants), within budgetary limits. ${ }^{23}$ This design gives 500 haplotypes at reasonable depth (that is, those transmitted to the children) and 500 haplotypes at half of that depth (that is, those not transmitted to the children). This design should significantly contribute to the globally existing information, as, for example, the 1000 Genomes Project has a $4 \mathrm{x}$ coverage. Paired-end sequencing of genomic DNA was done on the Illumina HiSeq 2000 platform (Illumina Inc., San Diego, CA, USA) at the Beijing Genomics Institute (BGI), starting in October 2010 and finished in June of 2011. Additionally, for each sample micro-array SNP data (Immunochip on all samples and at least one other GWAS array per sample) were generated by the biobanks. Sequencing was performed on 2-3 DNA libraries per sample which were run on 24 lanes of HiSeq 2000. The raw reads were subsequently analyzed following 1000 Genomes best practices, in collaboration with the Broad Institute and BGI. When reads were aligned against build 37 of the human genome coverage was, as expected, randomly Poisson distributed over the genome with a coverage peak in between 14-15x. The reads were cleaned by indel realignment, duplicate read marking and quality score recalibration, and SNP variants were called with a trio-aware caller. In parallel, a wide variety of short indels and complex structural variants were called and the SNPs were phased for imputation. Data processing involved $200000 \mathrm{~h}$ of computation and was run on the Dutch national Target and BigGrid compute facilities

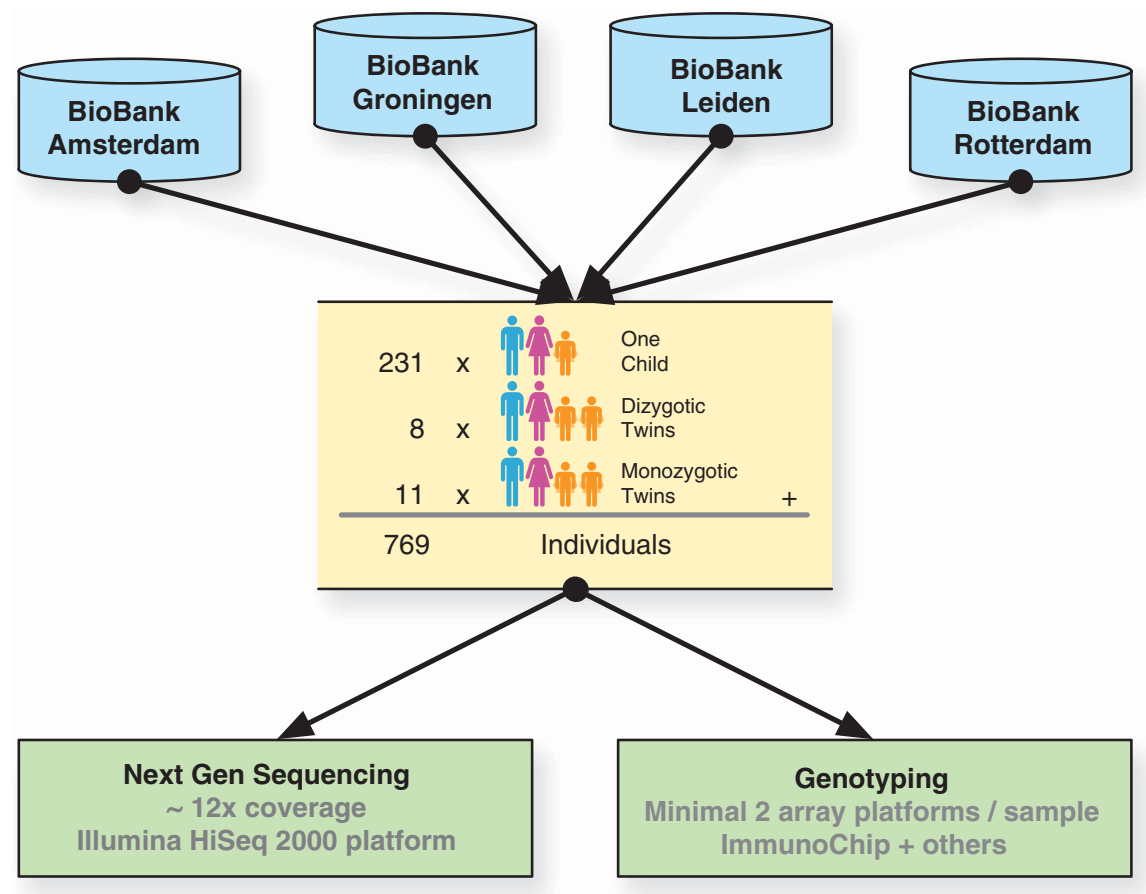

Figure 2 Sampling schedule for the GoNL: four population-based biobanks contributed samples for sequencing at the BGI (Beijing Genomics Institute). 
Table 2 Demographic characteristics and basic phenotypes for the GoNL participants. Values in brackets indicate ranges

\begin{tabular}{|c|c|c|c|c|c|c|c|c|}
\hline & Birth year & $\begin{array}{c}\text { Average } \\
\text { age at } \\
\text { sampling } \\
\text { (year) }\end{array}$ & $\begin{array}{c}\text { Average } \\
\text { height } \\
(\mathrm{cm})\end{array}$ & $\begin{array}{c}\text { Average } \\
\text { BMI }\left(\mathrm{kg} / \mathrm{m}^{2}\right)\end{array}$ & $\begin{array}{l}\text { Average } \\
\text { TC level } \\
\text { (mmol/l) }\end{array}$ & $\begin{array}{l}\text { Average } \\
\text { HDL level } \\
\text { (mmol/l) }\end{array}$ & $\begin{array}{l}\text { Average } \\
\text { LDL level } \\
\text { (mmol/l) }\end{array}$ & $\begin{array}{l}\text { Average } \\
\text { TG level } \\
\text { (mmol/l) }\end{array}$ \\
\hline Fathers $(N=250)$ & 1910-64 & $63.8(46-87)$ & $178(160-198)$ & $26.8(18.1-39.6)$ & $5.42(2.98-8.23)$ & $1.24(0.60-2.30)$ & $3.48(1.29-5.70)$ & $1.56(0.10-4.80)$ \\
\hline Mothers $(N=250)$ & 1910-64 & $61.7(43-86)$ & $166(145-182)$ & $27.0(18.6-38.9)$ & $5.67(3.10-8.70)$ & $1.51(0.55-2.46)$ & $3.54(1.48-6.40)$ & $1.35(0.16-4.65)$ \\
\hline
\end{tabular}
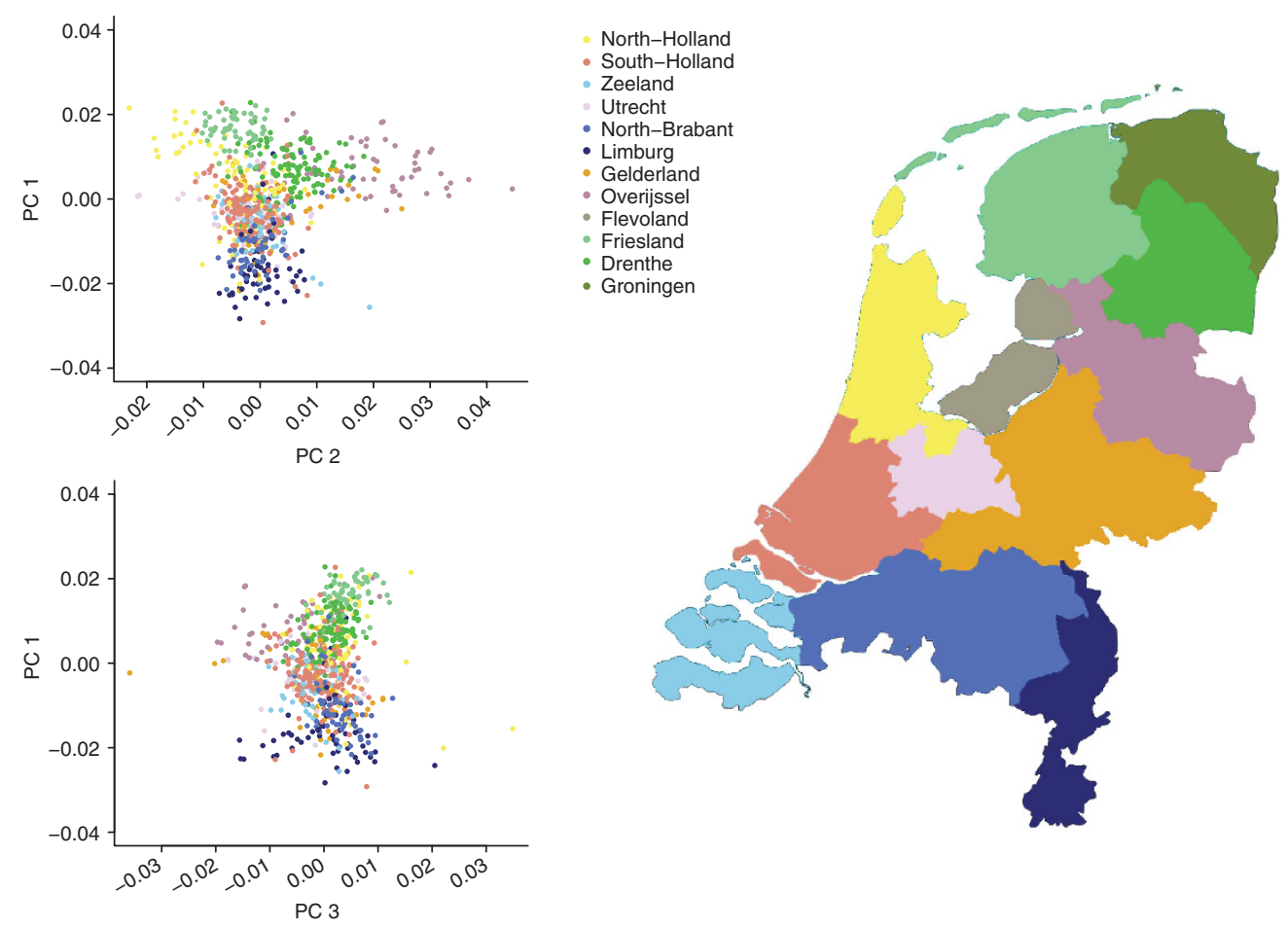

Figure 3 PCA results highlighting differences in genetic make-up across the Netherlands: the plots give PC1 versus PC2, and PC1 versus PC3.

supported by CIT/Groningen and SARA/Amsterdam. This effort resulted in $60 \mathrm{~TB}$ of aligned reads and variant calls (http:// www.rug.nl/target/infrastructuur, http://www.biggrid.nl/). While the sequencing technology and data processing pipelines are being improved continually, variant detection, including short indels and complex structural variants in low-complexity regions continue to pose a challenge. The GoNL data analysis group applied Genome Analysis Toolkit (GATK) BreakDancer, Pindel, ${ }^{24}$ GenomeSTRiP, CNVnator, 123SV and DWAC-seq to discover and genotype diverse types of variants such as SNPs, insertions, deletions, tandem duplications, inversions and translocations. ${ }^{25}$ Each of these tools showed its own variation detection characteristics, and the joint use of the tools turned out most powerful. Detailed analytical descriptions of SNP and SV calling, and de novo and loss-offunction variants will be reported separately. Detection of de novo mutations and accurate genotyping from pedigree data is challenging, especially at low or intermediate depth of coverage. Both require accurate and quantitative calibration of the evidence supporting the individuals' genotypes using all the available information, including their familial relationship and population allele frequency.

\section{Genomic structure of the Netherlands}

To describe the regional genomic differences in the genetic make-up across the Netherlands, principal component analysis (PCA) was run using the EIGENSOFT package. ${ }^{26}$ The PCA was first run on 4441 unrelated Dutch individuals ${ }^{15}$ typed on the Affymetrix Human Genome-Wide SNP 6.0 Array (Affymetrix Inc., Santa Clara, CA, USA), with a SNP set excluding 24 long-range LD regions ${ }^{27}$ and LDbased SNP pruning (based on a variance inflation factor of 2), resulting in a set of 130248 SNPs. These PCs were then projected on the 769 GoNL samples (using SNP calls release 2). Figure 3 summarizes the PC 1 versus PC 2 and the PC 1 versus PC 3 plots. Three PCs correlated significantly with geographic location and distinguished between: (1) the North and South of the Netherlands; (2) between the East and West; and (3) between the middle-band of the Netherlands and the rest of the country. When projected on the GoNL individuals, for whom we have the province of the current 
Table 3 Comparison of the designs of the 1000 Genomes and Genome of the Netherlands projects

\begin{tabular}{lll}
\hline & 1000G & GoNL \\
\hline Source of DNA & Cell lines & Blood \\
Coverage & $3-4 \times$ & $>12 \times$ \\
Data generation & Multiple centers/ platforms & BGI (Illumina HiSeq) \\
Population & Unrelated and multiple & One population (Dutch) and \\
& populations & trio/twin design \\
Phenotype information & None & Multiple, prospective \\
\hline
\end{tabular}

living address available, it is clear from Figure 3, that PCs capture the geographical variation in the data very well.

\section{CONCLUSION}

The goal of GoNL is to establish a reference set of variants in the Dutch human genome that have a frequency of at least $0.5 \%$ by whole-genome sequencing of 1000 independent genomes. Our pilot analyses reveal that new variants are likely to emerge compared with 1000 Genomes and may thus contribute to sequence data acquired by others. The GoNL project includes a number of unique aspects compared with other ongoing projects (see Table 3 ). The sequencing of GoNL was based on DNA isolated from blood (untransformed cells), whereas sequencing in 1000 Genomes involved DNA derived from transformed lymphoblastoid cell lines. Second, the embedding of the study in population-based prospective epidemiological cohorts enables research of clinical outcomes associated with mutations. Third, the trio design of the project in combination with the medium coverage of each genome allows estimation of haplotypes accurately, and to call a substantial amount of the more rare and de novo variation (both SNPs and structural variants). For 19 families, additional monzygotic or dizygotic twin siblings were sequenced, which allows in-depth research targeting new variants. Finally, the additional data on clinical phenotypes, epigenetics, RNA sequencing and the metabolome of the sequenced individuals will allow functional studies of new variants.

One of the main purposes is to catalog both common and rare variants, and to use the Dutch reference genome as a basis to computationally infer the corresponding information in a much larger data set of 100-150 thousand Dutch GWAS samples that have been generated in the Dutch population. The creation of an imputation pipeline is an important deliverable of GoNL. An imputation pipeline was created on the European Grid network for cohorts participating in BBMRI-NL, which can also be used by cohorts from elsewhere. The pipeline requires cohorts to upload their imputation-ready genotype data. Next, the process aligns the data with the Dutch and/or combined reference set and imputation will be done following recent protocols like the ones for the GIANT (the Genetic Investigation of ANthropometric Traits) consortium, using parallel grid computing. Afterwards, the full results including parameters for imputation quality can be downloaded by the cohort. The quality of imputation depends on several features of the input data and on the quality of the haplotype structure in the reference genome. The trio design with medium sequence coverage enables us to establish an accurately haplotyped Dutch reference genome, as the presence of Mendelian transmissions of variants provided an extra level of quality control and aided in the correct estimation of both genotypes and haplotypes.

The GoNL trio design allows calling a substantial amount of de novo variation (both SNPs and structural variants). It was recently shown that there is considerable variation in mutation rates within and between families, which in part can be attributed to the age of the father at the time of conception. ${ }^{28}$ With a cohort of 250 families, we will be able to further our understanding of mutational processes, in particular as GoNL also includes 19 families with two twin offspring. The twin pairs will allow us to address questions related to the developmental timing of de novo mutations and the frequency of germline mosaicism.

The GoNL data can also be used to address questions related to the demographic history of the Dutch as in-depth information on both mitochondrial $^{29}$ DNA and Y-chromosome will become available. Of immediate utility to the Dutch scientific community is the use of GoNL as a reference for clinical and medical-sequencing projects. Several founder mutations have previously been identified among the Dutch $^{16}$ including founder mutations in the BRCA1/2 (hereditary breast cancer), $L D L R$ (dyslipidemia) and Tau (dementia) genes. These findings predict that many genes may incorporate region and population-specific variants. The geographical variation observed in our preliminary analyses of substructure corroborates the importance of the original selection of GoNL samples dispersed throughout the Netherlands. The Dutch samples thus far included in multi-center GWAS studies originate from several different places in the Netherlands and may thus optimally benefit from imputation when the reference material properly reflects this provenance.

Within BBMRI-NL, several additional projects aim at harmonization and enrichment of biobanks within the Netherlands at a national scale. These projects include epigenetic studies, RNA sequencing and metabolomics projects. ${ }^{14}$ To fully understand the functional consequences of genetic variation, we have to integrate across these levels of information. The Dutch biobanks also contain a wealth of phenotypic information, which will become instrumental in pinpointing genes and genetic variants in disease. GoNL will be an excellent starting point to enrich the GWAS samples with more functional DNA variation, and with the BBMRI-NL infrastructure there is an excellent basis to further explain the functional consequences of disease-associated variants.

\section{Data access}

GoNL aims to be an open research source for researchers in the Netherlands and the rest of the world. The sequence data will be made available through $\mathrm{dbGaP}$ (http://www.ncbi.nlm.nih.gov/gap). Requests for data can be addressed to the steering committee of GoNL (see http://www.nlgenome.nl).

\section{CONFLICT OF INTEREST}

The authors declare no conflict of interest.

\section{ACKNOWLEDGEMENTS}

BBMRI-NL is a Research Infrastructure financed by the Dutch government (NWO 184.021.007).

\footnotetext{
1 Visscher PM, Brown MA, McCarthy MI, Yang J: Five years of GWAS discovery. Am J Hum Genet 2012; 90: 7.

2 So HC, Li M, Sham PC: Uncovering the total heritability explained by all true susceptibility variants in a genome-wide association study. Genet Epidemiol 2011; 35: 447-456.

3 Yang J, Benyamin B, McEvoy BP et al: Common SNPs explain a large proportion of the heritability for human height. Nat Genet 2010; 42: 565-569.

4 Yang J, Manolio TA, Pasquale LR et al: Genome partitioning of genetic variation for complex traits using common SNPs. Nat Genet 2011; 43: 519-525.
} 
5 Lubke GH, Hottenga JJ, Walters R et al: Estimating the genetic variance of major depressive disorder due to all single nucleotide polymorphisms. Biol Psychiatry 2012; 72: 707-709.

6 So HC, Gui AHS, Cherny SS, Sham PC: Evaluating the heritability explained by known susceptibility variants: a survey of ten complex diseases. Genet Epidemiol 2011; 35: 310-317.

7 Morris AP, Voight BF, Teslovich TM et al: Large-scale association analysis provides insights into the genetic architecture and pathophysiology of type 2 diabetes. Nat Genet 2012; 44: 981-990.

8 Naitza S, Porcu E, Steri M et al: A genome-wide association scan on the levels of markers of inflammation in Sardinians reveals associations that underpin its complex regulation. PLoS Genet 2012; 8: e1002480.

9 Rivas MA, Beaudoin M, Gardet A et al: Deep resequencing of GWAS loci identifies independent rare variants associated with inflammatory bowel disease. Nat Genet 2011; 43: 1066-1073.

10 Trynka G, Hunt KA, Bockett NA et al: Dense genotyping identifies and localizes multiple common and rare variant association signals in celiac disease. Nat Genet 2011; 43: 1193-1201.

11 Sanna S, Li B, Mulas A et al: Fine mapping of five loci associated with low-density lipoprotein cholesterol detects variants that double the explained heritability. PLoS Genet 2011; 7: e1002198.

12 Tennessen JA, Bigham AW, O'Connor TD et al: Evolution and functional impact of rare coding variation from deep sequencing of human exomes. Science 2012; 337: 64-69.

13 Nelson MR, Wegmann D, Ehm MG et al: An abundance of rare functional variants in 202 drug target genes sequenced in 14002 people. Science 2012; 337: 100-104.

14 Brandsma M, Baas F, de Bakker $\mathrm{P}$ et al: How to kickstart a national biobanking infrastructure-experiences and prospects of BBMRI-NL. Norsk Epidemiol 2012; 21: $143-148$.

15 Abdellaoui A, Hottenga JJ, de Knijff $\mathrm{P}$ et al: Population structure, migration, and diversifying selection in the Netherlands. Eur J Hum Genet 2013; 21: 1277-1285.

16 Zeegers MPA, Van Poppel F, Vlietinck R, Spruijt L, Ostrer H: Founder mutations among the Dutch. Eur J Hum Genet 2004; 12: 591-600.

17 Stolk RP, Rosmalen JGM, Postma DS et al: Universal risk factors for multifactorial diseases. Eur J Epidemiol 2008; 23: 67-74.
18 Schoenmaker M, de Craen AJM, de Meijer PHEM et al: Evidence of genetic enrichment for exceptional survival using a family approach: the Leiden Longevity Study. Eur J Hum Genet 2005; 14: 79-84.

19 van Beijsterveldt CEM, Groen-Blokhuis M, Hottenga JJ et al: The Young Netherlands Twin Register (YNTR): Longitudinal Twin and Family Studies in over 70000 children. Twin Res Hum Genet 2013; 16: 252-267.

20 Willemsen G, Vink JM, Abdellaoui A et al: The Adult Netherlands Twin Register: 25 years of survey and biological data collection. Twin Res Hum Genet 2013; 16: 271-281.

21 Willemsen G, de Geus EJC, Bartels M et al: The Netherlands Twin Register biobank: a resource for genetic epidemiological studies. Twin Res Hum Genet 2010; 13: 231.

22 Hofman A, van Duijn CM, Franco OH et al: The Rotterdam Study: 2012 objectives and design update. Eur J Epidemiol 2011; 26: 657-686.

23 Le SQ, Durbin R: SNP detection and genotyping from low-coverage sequencing data on multiple diploid samples. Genome Res 2011; 21: 952-960.

24 Ye K, Schulz MH, Long Q, Apweiler R, Ning Z: Pindel: a pattern growth approach to detect break points of large deletions and medium sized insertions from paired-end short reads. Bioinformatics 2009; 25: 2865-2871.

25 Guryev V, Kloosterman W, Francioli $L$ et al: Analysis of structural variation in the Genome of the Netherlands (GoNL) project. Eur J Hum Genet 2012; 20(Suppl 1): 23.

26 Price AL, Patterson NJ, Plenge RM, Weinblatt ME, Shadick NA, Reich D: Principal components analysis corrects for stratification in genome-wide association studies. Nat Genet 2006; 38: 904-909.

27 Price AL, Weale ME, Patterson $\mathrm{N}$ et al: Long-range LD can confound genome scans in admixed populations. Am J Hum Genet 2008; 83: 132-135.

28 Kong A, Frigge ML, Masson G et al: Rate of de novo mutations and the importance of father/'s age to disease risk. Nature 2012; 488: 471-475.

29 Vermaat M, Li M, den Dunnen J, de Knijff P, Stoneking M, Laros JFJ: Mitochondrial DNA analysis in the Genome of the Netherlands. Eur J Hum Genet 2012; 20(Suppl 1): 276.

This work is licensed under a Creative Commons Attribution 3.0 Unported License. To view a copy of this license, visit http://creativecommons.org/licenses/by/3.0/ 